International Journal of Pure and Applied Mathematics

Volume 84 No. 4 2013, 377-388

ISSN: 1311-8080 (printed version); ISSN: 1314-3395 (on-line version)

url: http://www.ijpam.eu

doi: http://dx.doi.org/10.12732/ijpam.v84i4.8

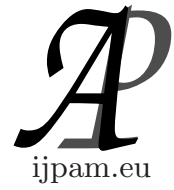

\title{
GENERALIZED HYERS-ULAM-RASSIAS THEOREM OF QUADRATIC FUNCTIONAL EQUATION IN MENGER PROBABLISTIC NORMED SPACES
}

\author{
V. Arasu ${ }^{1 \S}$, J.B. Stalin ${ }^{2}$ \\ ${ }^{1,2}$ Department of Mathematics \\ Adhiyamaan College of Engineering \\ Dr. M.G.R. Nagar, Hosur, 635 109, Tamil Nadu, INDIA
}

\begin{abstract}
In this paper, the authors investigate the generalized Hyers-UlamRassias stabitity of a quadratic functional equation $f(2 x+y)-4 f(x)-f(y)=$ $f(x+y)-f(x-y)$ in Menger Probablistic Normmed Spaces.
\end{abstract}

AMS Subject Classification: 39B22, 39B52, 39B82, $46 \mathrm{~S} 40$

Key Words: Hyers-Ulam-Rassias stability, quadratic functional equation, Menger probabilistic normed space

\section{Introduction}

In 1940, Ulam gave a talk before the Mathematics Club of the University of Wisconsin in which he discussed a number of unsolved problems. The stability problem of functional equations originated from a question of Ulam [27] concerning the stability of group homomorphisms.

In 1941, Hyers [8] considered the case of approximately additive functions $f: X \longrightarrow Y$ such that

$$
\|f(x+y)-f(x)-f(y)\| \leq \varepsilon
$$

for all $x, y \in X$ and for some $\varepsilon>0$, where $X$ and $Y$ are Banach spaces. Then

Received: December 5, 2012

(C) 2013 Academic Publications, Ltd.

${ }^{\S}$ Correspondence author 
there exists a unique additive function $A: X \longrightarrow Y$ such that

$$
\|f(x)-A(x)\| \leq \varepsilon
$$

for all $x \in X$.

Aoki [2] and Rassias [14] provided a generalization of the Hyers theorem for additive and linear functions, respectively, by allowing the Cauchy difference to be unbounded.

Theorem 1.1. Let $f: X \rightarrow Y$ be a function from a normed vector space $X$ into a Banach space $Y$ subject to the inequality

$$
\|f(x+y)-f(x)-f(y)\| \leq \varepsilon\left(\|x\|^{p}+\|y\|^{p}\right)
$$

for all $x, y \in X$, where $\varepsilon$ and $p$ are constants with $\varepsilon>0$ and $p<1$. Then there exists a unique additive function $A: X \rightarrow Y$ defined by $A(x)=\lim _{n \rightarrow \infty} 2^{-n} f\left(2^{n} x\right)$ is the unique additive mapping which satisfies

$$
\|f(x)-A(x)\| \leq \frac{2 \epsilon}{2-2^{p}}\|x\|^{p}
$$

for all $x \in X$. If $p<0$ then inequality (1.1) holds for $x, y \neq 0$ and (1.2) for $x \neq 0$. Also, if for each fixed $x \in X$ the function $t \mapsto f(t x)$ is continuous in $t \in \mathbb{R}$, then $A$ is linear.

The above Theorem has provided a lot of influence during the last three decades in the development of a generalization of the Hyers-Ulam stability concept. This new concept is known as generalized Hyers-Ulam stability or Hyers-Ulam-Rassias stability of functional equations (see $[4,9]$ ).

In 1994, a generalization of Rassias theorem was obtained by Gavruta [7] by replacing the bound $\epsilon\left(\|x\|^{p}+\|y\|^{p}\right)$ by a general control function $\varphi(x, y)$. Several stability results have been recently obtained for various equations, also for mapping with more general domains and ranges (see $[4,9,11]$ ).

During the last three decades a number of papers and research monographs have been published on various generalizations and applications of the generalized Hyers-Ulam stability to a number of functional equations and functions (see [3]-[10], [12, 13] and [17]-[23]).

Menger prposed the transferring some processes studied in applied sciences represented by differential equations. However, the situation is quite different in many physical phenomena that have a sudden change in their states such as mechanical systems with impact, biological systems such as heart beats, blood 
flows, population dynamics, theoretical physics, radiophysics, pharmacokinetics, mathematical economy, chemical technology, electric technology, metallurgy, ecology, industrial robotics, biotechnology, medicine and so on.

PN spaces were first defined and their definition was generalized by Serstnev in 1963 (see [26]). We recall and apply the definition of probabilistic space briefly as given in [24]

Definition 1.1. A Prpbabilistic Normed space (briefly, PN space) is a quadruple $(X, \nu, \tau, \tau *)$, where $X$ is a real vector space, $\tau$ and $\tau *$ are continuous triangle functions with $\tau \leq \tau *$ and $\nu$ is a mapping (the prpbabilistic norm) from $V$ into $\Delta^{+}$, such that for every choice of $p$ and $q$ in $V$ the following hold:

(N1) $\nu_{p}=\epsilon_{0}$ iff $p=\theta(\theta$ is the null vector in $\mathrm{X})$;

(N2) $\nu_{-p}=\nu_{p}$;

(N3) $\nu_{p+q} \geq \tau\left(\nu_{p}, \nu_{q}\right)$;

(N4) $\nu_{p} \leq \tau^{*}\left(\nu_{\lambda p}, \nu_{(1-\lambda) p}\right.$ for every $\lambda \in[0,1]$.

A PN space is called a Serstnev space if it satisfies (N1), (N3) and the following condition:

$$
\nu_{\alpha} p(x)=\nu_{p}\left(\frac{x}{|\alpha|}\right),
$$

holds for every $\alpha \neq 0 \in \Re$ and $x>0$. When $T$ is a continuos $t$-norm $T$ such that $\tau=\prod_{T}$ and $\tau^{*}=\prod_{T} *$, the PN space $(X, \nu, \tau, \tau *)$ is called Menger PN space (briefly, MPN space), and is denoted by $(X, \nu, \tau)$.

Let $(X, \nu, \tau)$ be an MPN space let $\left\{x_{n}\right\}$ be a sequence in $X$. Then $\left\{x_{n}\right\}$ is said to be convergent if there exists $x \in X$ such that

$$
\lim _{n \rightarrow \infty} \nu\left(x_{n}-x\right)(t)=1
$$

for all $t>0$. In this case $x$ is called the limit of $\left\{x_{n}\right\}$.

The sequence $x_{n}$ in MPN space $(X, \nu, \tau)$ is called Cauchy if for each $\epsilon>0$ and $\delta>0$, there exists some $n_{0}$ such that $\nu\left(x_{n}-x_{m}\right)(\delta)>1-\epsilon$ for all $m, n \geq n_{0}$.

Clearly, every convergent sequence in a MPN sapce is Cauchy. If eqch Cauchy sequence is convergent in a MPN space $(X, \nu, \tau)$, then $(X, \nu, \tau)$ is called Menger Probabiistic Banach space (briefly, MPB space). 
Recently, the stability of functional equations on PN spaces and MPN spaces have been investigated by some authors; (see $[6,25]$ ). and refrences therein. In this paper, we invetigate the stability of qudratic functional equations on Serstnev probabilistic normed space endowed with $\prod_{M}$ triangle function.

\section{Main Results}

We begin our work with uniform version of the Hyers-Ulam-Rassias stability in a Serstnev PN space in which we uniformly approximate a uniform quadratic mapping.

Theorem 2.1. Let $X$ be a linear space and $\left(\gamma, \nu, \prod_{M}\right)$ be a Serstnev PB space. Let $\varphi: X \times X \rightarrow[0, \infty)$ be a control function such that

$$
\widetilde{\varphi_{n}}(x, 0)=\left\{4^{-n-1} \varphi\left(2^{n} x, 0\right)\right\} \quad(x \in X)
$$

converges to zero. Let $f: X \rightarrow \gamma$ be a uniformly approximately quadratic function with respect to $\varphi$ in the sense that

$$
\lim _{t \rightarrow \infty} \nu(f(2 x+y)-4 f(x)-f(y)-f(x+y)+f(x-y))(t \varphi(x, y))=1
$$

uniformly on $X \times X$. Then $T(x):=\lim _{n \rightarrow \infty} 2^{-n} f\left(2^{n} x\right)$ for any $x \in X$ exists and defines an additive mapping $T: X \rightarrow \gamma$ such that if for some $\delta>0, \alpha>0$

$\nu(f(2 x+y)-4 f(x)-f(y)-f(x+y)+f(x-y))(\delta \varphi(x, 0))>\alpha \quad(x, y \in X)$

then

$$
\nu(T(x)-f(x))\left(\delta \widetilde{\varphi_{n}}(x, 0)\right)>\alpha \quad(x \in X) .
$$

Proof. Given $\epsilon>0$, by $(2.2)$ we can choose some $t_{0}$ such that

$$
\nu(f(2 x+y)-4 f(x)-f(y)-f(x+y)+f(x-y))(t \varphi(x, 0))>1-\epsilon
$$

for all $x, y \in X$ and all $t \geq t_{0}$. Subsituting $y=0$ in (2.5) we obtain

$$
\nu(f(2 x)-4 f(x))(t \varphi(x, 0))>1-\epsilon
$$

and replacing $x$ by $2^{n} x$, we get

$$
\nu\left(4^{-n-1} f\left(2^{n+1} x\right)-4^{-n} f\left(2^{n} x\right)\right)\left(t 4^{-n-1} \varphi\left(2^{n} x, 0\right)\right)>1-\epsilon .
$$


Allowing to a nonincreasing subequence, if necessary, we assume that

$$
\left\{4^{-n-1} \varphi\left(2^{n} x, 0\right)\right\}
$$

is nonincreasing.

Thus for each $n>m$ we have

$$
\begin{aligned}
\nu\left(4^{-m} f\left(2^{m} x\right)-4^{-n} f\left(2^{n} x\right)\right)\left(t 4^{-m-1} \varphi\left(2^{m} x, 0\right)\right) \\
=\nu \sum_{k=m}^{n-1}\left(4^{-k} f\left(2^{k} x\right)-4^{-k-1} f\left(2^{k+1} x\right)\right)\left(t 4^{-m-1} \varphi\left(2^{m} x, 0\right)\right) \\
\geq \prod_{M}\left(\nu\left(4^{-m} f\left(2^{m} x\right)-4^{-m-1} f\left(2^{m+1} x\right)\right),\right. \\
\quad \nu\left(\sum_{k=m+1}^{n-1}\left(4^{-k} f\left(2^{k} x\right)-4^{-k-1} f\left(2^{k+1} x\right)\right)\right)\left(t 4^{-m-1} \varphi\left(2^{m} x, 0\right)\right) \\
\geq \prod_{M}\left(1-\epsilon ; \prod_{M}\left(\left(\nu\left(4^{-m-1} f\left(2^{m+1} x\right)-4^{-m-2} f\left(2^{m+2} x\right)\right),\right.\right.\right. \\
\left.\quad \nu\left(\sum_{k=m+2}^{n-1}\left(4^{-k} f\left(2^{k} x\right)-4^{-k-1} f\left(2^{k+1} x\right)\right)\right)\left(t 4^{-m-2} \varphi\left(2^{m+1} x, 0\right)\right)\right) \\
\geq 1-\epsilon .
\end{aligned}
$$

The convergence of (2.1) implies that for given $\delta>0$ there is $n_{0} \in N$ such that

$$
t_{0} 4^{-n-1} \varphi\left(2^{n} x, 0\right)<\delta \quad \forall n \geq n_{0} .
$$

Thus by (2.8) we deduce that

$$
\begin{aligned}
& \nu\left(4^{-m} f\left(2^{m} x\right)-4^{-n} f\left(2^{n} x\right)\right)(\delta) \\
& \quad \geq \nu\left(4^{-m} f\left(2^{m} x\right)-4^{-n} f\left(2^{n} x\right)\right)\left(t_{0} 4^{-m-1} \varphi\left(2^{m} x, 0\right)\right) \geq 1-\epsilon
\end{aligned}
$$

for each $n \geq n_{0}$. Hence $4^{-n} f\left(2^{n} x\right)$ is a Cauchy sequence in $\gamma$. Since $\left(\gamma, \nu, \prod_{M}\right)$ is complete,this sequence converges to some $T(x) \in \gamma$. Therefore we can define a mapping $T: X \rightarrow \gamma$ by $T(x):=\lim _{n \rightarrow \infty} 4^{-n} f\left(2^{n} x\right)$, namely,for each $t>0$, and $x \in X$,

$$
\nu\left(T(x)-4^{-n} f\left(2^{n} x\right)\right)(t)=1 .
$$

Next, let $x, y \in X$. Temporarily fix $t>0$ and $0<\epsilon<1$. Since $4^{-n-1} \varphi\left(2^{n} x, 0\right)$ converges to zero, there is some $n_{1}>n_{0}$ such that $t_{0} \varphi\left(2^{n} x, 0\right)<t 2^{n+1}$ for all $n>n_{1}$, we have

$$
\nu(T(2 x+y)-4 T(x)-T(y)-T(x+y)+T(x-y))(t)
$$




$$
\begin{aligned}
& \geq \prod_{M}\left(\prod _ { M } \left(\nu\left(T(2 x+y)-4^{-n-1} f\left(2^{n+1}(2 x+y)\right)\right)(t), \nu 4(T(x)\right.\right. \\
& \left.\left.-4^{n-1} f\left(2^{n+1} x\right)\right)(t)\right) \\
& \prod_{M}\left(\nu\left(T(y)-4^{n-1} f\left(2^{n+1} y\right)\right)(t), \nu\left(T(x+y)-4^{-n-1} f\left(2^{n+1}(x+y)\right)\right)(t)\right) \\
& \prod_{M}\left(\nu\left(T(x-y)-4^{-n-1} f\left(2^{n+1}(x-y)\right)\right)(t)\right. \\
& \nu\left(f\left(2^{n+1}(2 x+y)\right)-4 f\left(2^{n+1} x\right)-f\left(2^{n+1} y\right)-f\left(2^{n+1}(x+y)\right)\right. \\
& \left.\left.\left.+\left(2^{n+1}(x-y)\right)\right)\left(4^{n+1} t\right)\right)\right)
\end{aligned}
$$

we have

$$
\begin{array}{r}
\lim _{n \rightarrow \infty} \nu\left(T(2 x+y)-4^{-n-1} f\left(2^{n+1}(2 x+y)\right)\right)(t)=1, \\
\lim _{n \rightarrow \infty} \nu\left(T(x)-4^{-n-1} f\left(2^{n+1} x\right)\right)(t)=1, \\
\lim _{n \rightarrow \infty} \nu\left(T(y)-4^{-n-1} f\left(2^{n+1} y\right)\right)(t)=1, \\
\lim _{n \rightarrow \infty} \nu\left(T(x+y)-4^{-n-1} f\left(2^{n+1}(x+y)\right)\right)(t)=1, \\
\lim _{n \rightarrow \infty} \nu\left(T(x-y)-4^{-n-1} f\left(2^{n+1}(x-y)\right)\right)(t)=1,
\end{array}
$$

and by (2.5) and large enough $n$, we have

$$
\begin{aligned}
& \nu\left(f\left(2^{n+1}(2 x+y)\right)-4 f\left(2^{n+1} x\right)-f\left(2^{n+1} y\right)-f\left(2^{n+1}(x+y)\right)\right. \\
& \left.\quad+f\left(2^{n+1}(x-y)\right)\right)\left(4^{n+1} t\right) \\
& \geq \nu\left(f\left(2^{n+1}(2 x+y)\right)-4 f\left(2^{n+1} x\right)-f\left(2^{n+1} y\right)\right. \\
& \quad-f\left(2^{n+1}(x+y) t\right)+f\left(2^{n+1}(x-y)\right)\left(t_{0} \varphi\left(2^{n} x, 0\right)\right) \geq 1-\epsilon .
\end{aligned}
$$

Thus

$$
\begin{aligned}
& \nu(T(2 x+y)-4 T(x)-T(y)-T(x+y)+T(x-y))(t) \geq 1-\epsilon, \\
& \forall t>0,0<\epsilon<1 .
\end{aligned}
$$

To end the proof, let us take for some positive $\delta$ and $\alpha,(2.3)$ holds. Let $x \in X$. Setting $m=0$ and $\alpha=1-\epsilon$ in (2.10), we get

$$
\nu\left(f\left(2^{n} x\right)-4^{n} f(x)\right)(\delta) \geq \alpha
$$

for all positive integers $n \geq n_{0}$. For large enough $n$, we have $\nu(f(x)-T(x))\left(\delta 4^{-n-1} \varphi\left(2^{n} x, 0\right)\right.$ 


$$
\geq \prod_{M}\left\{\nu\left(f(x)-4^{-n} f\left(2^{n} x\right)\right), \nu\left(4^{-n} f\left(2^{n} x\right)-T(x)\right)\right\}\left(\delta 4^{-n-1} \varphi\left(2^{n} x, 0\right)\right) \geq \alpha
$$

which implies

$$
\nu(T(x)-f(x))\left(\delta \widetilde{\varphi_{n}}(x, 0)\right)>\alpha .
$$

Corollary 2.1. Let $X$ be a linear space defined by (2.1) and $\left(\gamma, \nu, \prod_{M}\right)$ a Serstnev $P B$ space Let $\varphi: X \times X \rightarrow[0, \infty)$ be a control function satisfying (2.2). Let $f: X \rightarrow \gamma$ be a uniformly approximately quadratic function with respect to $\varphi$. Then there is a unique quadratic mapping $T: X \rightarrow \gamma$ such that

$$
\lim _{n \rightarrow \infty} \nu(f(x)-T(x))\left(t \widetilde{\varphi_{n}}(x, 0)\right)
$$

uniformly on $X$.

Proof. The existence of uniform limit (2.19) immediately follows from Theorem (2.1). It remans to prove the uniqueness assertion.

Let $S$ be another quadratic mapping satisfying (2.19). Fix $c>0$. Given $\epsilon>0$, by (2.19) for $T$ and $S$,we can find some $t_{0}>0$ such that

$$
\begin{aligned}
& \nu(f(x)-T(x))\left(t \widetilde{\varphi_{n}}(x, 0)\right)>1-\epsilon, \\
& \nu(f(x)-S(x))\left(t \widetilde{\varphi_{n}}(x, 0)\right)>1-\epsilon .
\end{aligned}
$$

for all $x \in X$ and $t \geq t_{0}$. Fix for some $x \in X$ and find some integer $n_{0}$ such that

$$
t_{0} 4^{-n} \varphi\left(2^{n+1} x, 0\right)>c \forall n \geq n_{0}
$$

Then we have

$$
\begin{aligned}
\nu(S(x) & -T(x))(c) \\
& \geq \prod_{M}\left\{\nu\left(4^{-n} f\left(2^{n} x\right)-T(x)\right), \nu\left(S(x)-4^{-n} f\left(2^{n} x\right)\right)\right\}(c) \\
& =\prod_{M}\left\{\nu\left(f\left(2^{n} x\right)-T\left(2^{n} x\right)\right), \nu\left(S\left(2^{n} x\right)-f\left(2^{n} x\right)\right)\right\}\left(2^{n} c\right) \\
& \geq \prod_{M}\left\{\nu\left(f\left(2^{n} x\right)-T\left(2^{n} x\right)\right), \nu\left(S\left(2^{n} x\right)-f\left(2^{n} x\right)\right)\right\}\left(2^{n} c\right)\left(t_{0} 4^{-n} \varphi\left(2^{n+1} x, 0\right)\right) \\
& \geq 1-\epsilon .
\end{aligned}
$$

It follows that $\nu(S(x)-T(x))(c)=1$ for all $c>0$. Thus $T(x)=S(x)$ for all $x \in X$. 
Theorem 2.2. Let $X$ be a linear space. Let $\left(Z, \omega, \prod_{M}\right)$ be a Serstnev MPN space. Let $\psi: X \times X \rightarrow Z$ be a function such that for all $0<\alpha<4$,

$$
\omega(\psi(2 x, 0))(t) \geq \omega(\psi(x, 0))(t)
$$

for all $x, y \in X$ and $t>0$. Let $\left(\gamma, \nu, \prod_{M}\right)$ be a Serstnev $P B$ space and let $f: X \rightarrow \gamma$ be a $\psi$ approximately quadratic mapping in the sense that

$$
\nu(f(2 x+y)-4 f(x)-f(y)-f(x+y)+f(x-y))(t) \geq \omega(\psi(x, 0))(t)
$$

for eqch $t>0$ and $x, y \in X$. Then there exists unique quadratic mapping $T: X \rightarrow \gamma$ such that

$$
\nu(f(x)-T(x))(t) \geq \omega\left(\frac{1}{4} \psi(x, 0)(t)\right)
$$

where $x \in X$ and $t>0$.

Proof. Put $y=0$ in (2.24) we get

$$
\nu(f(2 x)-4 f(x))(t) \geq \omega(\psi(x, 0))(t) \quad(x \in X, t>0) .
$$

Using (2.23) and using induction on $n$, we obtain

$$
\omega\left(\psi\left(2^{n} x, 0\right)\right)(t) \geq \omega\left(\alpha^{n}(x, 0)\right)(t)
$$

for all $x \in X$ and $t>0$. Replacing $x$ by $4^{n-1} x$ in (2.26) and using (2.27) we get

$$
\nu\left(f\left(2^{n} x\right)-4 f\left(2^{n-1} x\right)\right)(t) \geq \omega\left(\left(\alpha^{n-1} \psi(x, 0)\right)(t)\right.
$$

for all $x \in X$ and $t>0$. It follows from (2.28), we get

$$
\nu\left(4^{-n} f\left(2^{n} x\right)-4^{-n+1} f\left(2^{n-1} x\right)\right)\left(4^{-n} t\right) \geq \omega\left(\left(\frac{1}{\alpha}\right) \psi(x, 0)\right)\left(\alpha^{-n} t\right)
$$

whence

$$
\nu\left(4^{-n} f\left(2^{n} x\right)-4^{-n+1} f\left(2^{n-1} x\right)\right)\left(\left(\frac{\alpha^{n}}{4^{n}}\right) t\right) \geq \omega\left(\frac{1}{\alpha} \psi(x, 0)\right)(t),
$$

for all $n>m \geq 0, x \in X$ and $t>0$. So

$$
\nu\left(4^{-n} f\left(2^{n} x\right)-4^{-m} f\left(2^{m} x\right)\right)\left(\left(\frac{\alpha^{m+1}}{4^{m+1}}\right) t\right)
$$




$$
\begin{aligned}
& =\nu\left(\sum_{n}^{k=m+1} 4^{-k} f\left(2^{k} x\right)-4^{-k+1} f\left(2^{k-1} x\right)\right)\left(\left(\frac{\alpha^{m+1}}{4^{m+1}}\right) t\right) \\
& \geq \omega\left(\frac{1}{\alpha} \psi(x, 0)\right)(t)
\end{aligned}
$$

whence

$$
\nu\left(4^{-n} f\left(2^{n} x\right)-4^{-m} f\left(2^{m} x\right)\right)(t) \geq \omega\left(\left(\frac{1}{\alpha}\right) \psi(x, 0)\right)\left(\left(\frac{\alpha^{m+1}}{4^{m+1}}\right) t\right)
$$

for all $n>m \geq 0, x \in X$ and $t>0$.Fix $x \in X$. By

$$
\lim _{s \rightarrow \infty} \omega\left(\frac{1}{\alpha} \psi(x, 0)\right)(s)=1
$$

we reduce that $4^{-n} f\left(2^{n} x\right)$ is a Cauchy sequence in $\left(\gamma, \nu, \prod_{M}\right)$. Since $\left(\gamma, \nu, \prod_{M}\right)$ is complete, this sequence converges to some point $T(x) \in \gamma$. It follows from (2.24) that

$$
\begin{aligned}
\nu\left(f\left(2^{n}(2 x+y)\right)-4 f\left(2^{n} x\right)-\right. & f\left(2^{n} y\right)-f\left(2^{n}(x+y)\right)+f\left(2^{n}(x-y)\right)(t) \\
& \geq \omega\left(\psi\left(2^{n} x, 2^{n} y\right)\right)(t) \\
& \geq \omega\left(\alpha^{n} \psi(x, y)\right)(t) \\
& \geq \omega(\psi(x, y))\left(\alpha^{-n} t\right)
\end{aligned}
$$

whence

$$
\begin{aligned}
\nu\left(4^{-n} f\left(2^{n}(2 x+y)\right)\right. & -4^{-n} 4 f\left(2^{n} x\right)-4^{-n} f\left(2^{n} y\right)-4^{-n} f\left(2^{n}(x+y)\right) \\
& \left.+4^{-n} f\left(2^{n}(x-y)\right)\right)(t) \geq \omega(\psi(x, y))\left(\left(\frac{4}{\alpha}\right)^{n} t\right)
\end{aligned}
$$

we have

$$
\begin{gathered}
\nu(T(2 x+y)-4 T(x)-T(y)-T(x+y)+T(x-y))(t) \\
\geq \prod_{M}\left(\prod _ { M } \left\{\nu\left(T(2 x+y)-4^{-n} f\left(2^{n}(2 x+y)\right)\right), \nu\left(4\left(T(x)-4^{-n} f\left(2^{n} x\right)\right)\right)(t),\right.\right. \\
\prod_{M}\left(\nu\left(T(y)-4^{-n} f\left(2^{n} y\right)\right), \nu\left(T(x+y)-4^{-n} f\left(2^{n}(x+y)\right)\right)(t)\right), \\
\prod_{M}\left(\nu\left(T(x-y)-4^{-n} f\left(2^{n}(x-y)\right)\right), \nu\left(4^{-n} f\left(2^{n}(2 x+y)\right)-\right.\right. \\
\left.\left.4^{-n} f\left(2^{n} x\right)-4^{-n} f\left(2^{n} y\right)-4^{-n} f\left(2^{n}(x+y)\right)+4^{-n}\left(2^{n}(x-y)\right)\right)\right)(t) .
\end{gathered}
$$


By (2.35) and the fact that

$$
\lim _{n \rightarrow \infty} \nu\left(T(z)-4^{-n} f\left(2^{n} z\right)\right)=1
$$

for all $z \in X$ and $r>0$, each term on the rignt-hand side tends to 1 as $n \rightarrow \infty$. Hence

$$
\nu(T(2 x+y)-4 T(x)-T(y)-T(x+y)+T(x-y))=1 .
$$

By (N1), it means that

$$
T(2 x+y)-4 T(x)-T(y)=T(x+y)-T(x-y)
$$

Furthermore, let $x \in X$ and $t>0$. Using (2.32) with $m=0$ we get

$$
\begin{aligned}
\nu(T(x) & -f(x))(t) \\
& \geq \prod_{M}\left\{\nu\left(T(x)-4^{-n} f\left(2^{n} x\right), \nu\left(4^{-n} f\left(2^{n} x\right)-f(x)\right)\right\}(t)\right. \\
& \geq \prod_{M}\left\{\nu\left(T(x)-4^{-n} f\left(2^{n} x\right), \omega\left(\frac{1}{4} \psi(x, 0)\right)\right\}(t) .\right.
\end{aligned}
$$

Hence

$$
\begin{aligned}
\nu(T(x) & -f(x))(t) \\
& \geq \prod_{M}\left\{\nu\left(T(x)-4^{-n} f\left(2^{n} x\right), \omega\left(\frac{1}{4} \psi(x, 0)\right)\right\}(t)\right. \\
& \geq \omega\left(\frac{1}{4} \psi(x, 0)\right)(t) .
\end{aligned}
$$

The uniqueness of $T$ can be proved in a similar manner as in the proof of corollary (2.1).

\section{References}

[1] J. Aczel, J. Dhombres, Functional Equations in Several Variables, Cambridge University Press, 1989.

[2] T. Aoki, On the stability of the linear transformation in Banach spaces, $J$. Math. Soc. Japan., 2 (1950), 64-66. 
[3] P.W. Cholewa, Remarks on the stability of functional equations, Aequationes Math., 27 (1984), 76-86.

[4] P. Czerwik, Functional Equations and Inequalities in Several Variables, World Scientific Publishing Company, New Jersey, Hong Kong, Singapore, London, 2002.

[5] S. Czerwik, On the stability of the quadratic mapping in normed spaces, Abh. Math. Sem. Univ. Hamburg., 62 (1992), 59-64.

[6] M. Eshaghi Gordji and M.B. Savadkouchi, Approximation of the Quadratic and Cubic Functional Equations in RN-spaces, European Journal of Pure and Applied Mathematics, 2 (2009), 494-507.

[7] P. Găvruta, A generalization of the Hyers-Ulam-Rassias stability of approximately additive mappings, J. Math. Anal. Appl., 184 (1994), 431-436.

[8] D.H. Hyers, On the stability of the linear functional equation, Proc. Natl. Acad. Sci., 27 (1941), 222-224.

[9] D.H. Hyers, G. Isac, Th.M. Rassias, Stability of Functional Equations in Several Variables, Birkhäuser, Basel, 1998.

[10] G. Isac, Th.M. Rassias, Stability of $\psi$-additive mappings: Applications to nonlinear analysis, Internat. J. Math. Math. Sci., 19 (1996), 219-228.

[11] S.-M. Jung, Hyers-Ulam-Rassias Stability of Functional Equations in Mathematical Analysis, Hadronic Press Inc., Palm Harbor, Florida, 2001.

[12] S.-M. Jung, T.-S. Kim, A fixed point approach to stability of cubic functional equation, Bol. Soc. Mat. Mexicana, 12 (2006), 51-57.

[13] Pl. Kannappan, Quadratic functional equation and inner product spaces, Results Math., 27 (1995), 368-372.

[14] Th.M. Rassias, On the stability of the linear mapping in Banach spaces, Proc. Amer. Math. Soc., 72 (1978), 297-300.

[15] Th.M. Rassias, Functional Equations, Inequalities and Applications, Kluwer Academic Publishers Co., Dordrecht, Boston, London, 2003.

[16] Th.M. Rassias, Problem 16; 2, Report of the 2rth International Symp. on Functional Equations, Aequationes Math., 39 (1990), 292-293. 
[17] Th.M. Rassias, On the stability of the quadratic functional equation and its applications, Studia Univ. Babes-Bolyai., XLIII (1998), 89-124.

[18] Th.M. Rassias, The problem of S.M. Ulam for approximately multiplicative mappings, J. Math. Anal. Appl., 246 (2000), 352-378.

[19] Th.M. Rassias, On the stability of functional equations in Banach spaces, J. Math. Anal. Appl., 251 (2000), 264-284.

[20] Th.M. Rassias, On the stability of functional equations and a problem of Ulam, Acta Appl. Math., 62 (2000), 23-130.

[21] Th.M. Rassias, P. Šemrl, On the behaviour of mappings which do not satisfy Hyers-Ulam stability, Proc. Amer. Math. Soc., 114 (1992), 989993.

[22] Th.M. Rassias and P. Šemrl, On the Hyers-Ulam stability of linear mappings, J. Math. Anal. Appl., 173 (1993), 325-338.

[23] Th.M. Rassias, K. Shibata, Variational problem of some quadratic functionals in complex analysis, J. Math. Anal. Appl., 228 (1998), 234-253.

[24] B. Schweizer, A. Sklar, Probabilistic Metric Spaces, Elsevier, North Holand, New York, 1983.

[25] S. Shakeri, R. Saadati, Gh. Sadeghi, S.M. Vaezpour, Stability of the Cubic Functional Equation in Menger Probabilistic Normed Spaces, Journal of Apllied Sciences, 9 (2009), 1795-1797.

[26] A.N. Šerstnev, On the notion of a random normed space, Dokl. Akad. Nauk SSSR, 149 (1963), 280-283, In Russian.

[27] S.M. Ulam, Problems in Modern Mathematics, Chapter VI, science Editions., Wiley, New York, 1964. 